\title{
Multiname and Multiscale Default Modeling
}

\author{
Jean-Pierre Fouque* Ronnie Sircar ${ }^{\dagger} \quad$ Knut Sølna ${ }^{\ddagger}$
}

June 9,2008

\begin{abstract}
Multiname default modeling is crucial in the context of pricing credit derivatives such as Collaterized Debt Obligations (CDOs). We consider here a simple reduced form approach for multiname defaults based on the Vasicek or Ornstein-Uhlenbeck model for the hazard rates of the underlying names. We analyze the impact of volatility time scales on the default distribution and CDO prices. We demonstrate how correlated fluctuations in the parameters of the name hazard rates affect the loss distribution and senior tranches of CDOs. The effect of stochastic parameter fluctuations is to change the shape of the loss distribution and cannot be captured by using averaged parameters in the original model. Our analysis assumes a separation of time scales and leads to a singular-regular perturbation problem $[7,8]$. This framework allows us to compute perturbation approximations that can be used for effective pricing of CDOs.
\end{abstract}

\section{Introduction}

Credit derivatives are financial securities that pay their holders amounts that are contingent on the occurrence (or not) of one or more default events such as the bankruptcy of a firm or nonrepayment of a loan. The dramatic losses in the credit derivatives market in 2007 illustrates that the problem of appropriate modeling and pricing of large portfolios of debt obligations is challenging and also a largely open question. The mathematical challenge is to model the default times of the firms (or names) and, most importantly, the correlation between them. Part of the challenge is that incorporating heterogeneity and correlations may appear as intractable due to the curse of combinatorial complexity. Here we consider pricing of collateralized debt obligations (CDOs) using intensity-based models with multiscale stochastic volatility. A main aspect of our approach is to make use of approximation methods via singular and regular perturbation expansions that make the multi-dimensional problems tractable.

In the last few years, the standard in the industry has been copulas for creating correlation structures. This is even the case at present after the recent credit crisis. The main drawback of this approach is the fact that these are static models which do not take into account the time evolution of joint default risks. This has been recognized in the academic literature on dynamic models with recent developments in the multiname structural approach [10,13], reduced form models [2, 16, 15, 4], and top-down models $[6,14,18]$.

\footnotetext{
*Department of Statistics and Applied Probability, University of California, Santa Barbara, CA 93106-3110, fouque@pstat.ucsb.edu. Work partially supported by NSF grant DMS-0455982.

${ }^{\dagger}$ Department of Operations Research \& Financial Engineering, Princeton University, E-Quad, Princeton, NJ 08544, sircar@princeton.edu. Work partially supported by NSF grant DMS-0456195.

${ }^{\ddagger}$ Department of Mathematics, University of California, Irvine CA 92697, ksolna@math.uci.edu.
} 
We shall consider the case when we use the Vasicek model in the context of (bottom-up) multiname reduced form modeling of credit risk. Here, we let the hazard rates of the default times of the $N$ names be specified as correlated Vasicek or Ornstein-Uhlenbeck processes. Clearly, in this Gaussian model, the intensity might become negative. But, as stated in [3], "the computational advantage with explicit solutions may be worth the approximation error associated with this Gaussian formulation". This is particularly the case when it comes to calibration, which typically involves a large number of evaluations of the default probabilities as part of an iterative procedure. In addition to "explicitly modeled" correlation in between name hazard rates, implicit correlation generated by a common factor is important. This phenomenon, with a common volatility factor has been analyzed in $[9,10,16]$ and we continue here this line of research. We show below how the roles of "explicit and implicit" correlations in joint default probabilities become transparent in the Vasicek framework.

We introduce the pricing problem in Section 2.1 and discuss the doubly stochastic framework in some more detail in Section 2.2. See for instance $[3,17]$ for a detailed discussion of these concepts. In Section 2.3 we discuss the Vasicek credit model and pricing of CDOs. Our focus is on correlation effects between names and the effects of stochastic multiscale parameter variations. We discuss both the symmetric case with simple explicit formulas for survival probabilities and the case where there is heterogeneity in between the models for the name intensities. In the latter case effective computational procedures via conditioning are given and we illustrate with numerical examples.

\section{Modeling}

\subsection{The CDO contract}

CDOs are designed to securitise portfolios of defaultable assets. Their main feature is that the total nominal associated with the names or obligors is sliced into tranches. Each tranche is then insured against default. The first default events apply to the first tranche and so on. The protection seller for the first tranche, the equity tranche, is therefore strongly exposed to credit risk relative to the protection sellers for the subsequent mezzanine and senior/super-senior tranches, and the CDO provides a prioritization of credit risk. Two credit derivative indexes are the US based CDX and the European iTraxx. Each tranche is described by a lower and an upper attachment point. In the CDX case the decomposition into tranches corresponds to $\{0-3,3-7,7-10,10-15,15-30\} \%$ of the total nominal and this is the decomposition we shall use below in our computational examples.

Let $\alpha_{\ell}$ be the yield associated with tranche $\ell$, that is, the rate at which the insurance buyer pays for protection of tranche $\ell$. In the event of default in this tranche, the protection seller pays a fraction $1-R$ of the loss, with $R$ being the recovery, to the tranche holder (the buyer). We shall assume a fixed recovery, and, moreover, that the payments are made at a set of predetermined times $T_{k}, k \in\{1,2, \cdots, K\}$. Under the pricing measure $\mathbb{P}^{\star}$, we have that $\alpha_{\ell}$ is determined by:

$$
\sum_{k} e^{-r T_{k}} f_{\ell}\left(T_{k-1}\right) \alpha_{\ell}\left(T_{k}-T_{k-1}\right)=\sum_{k} e^{-r T_{k}}\left(f_{\ell}\left(T_{k-1}\right)-f_{\ell}\left(T_{k}\right)\right)(1-R)
$$

where $r$ is the constant short rate and $f_{\ell}\left(T_{k}\right)$ is the expected fraction of tranche $\ell$ left at time $T_{k}$. This is an approximation corresponding to the defaults occurring in the time interval from $T_{k-1}$ to $T_{k}$ being accounted at the end of this time interval. This is the simple model contract we use below when the tranches are those associated with the CDX. Further details can be found in [5], for instance.

In the following, we shall compute the tranche prices $\alpha_{\ell}$ when the default of the obligors are modeled in terms of the Vasicek reduced form model, and we comment next on the doubly stochastic modeling. 


\subsection{Doubly Stochastic Modeling}

We consider $N$ obligors or underlying names. The event that a particular obligor $i$ defaults is modeled in terms of the first arrival $\tau_{i}$ of a Cox process with stochastic intensity or hazard rate $X^{(i)}$. Conditioned on the paths of the hazard rates, the default times $\tau_{i}$ of the firms are independent, and the probability that obligor $i$ has survived till time $T$, is given by $\exp \left(-\int_{0}^{T} X_{s}^{(i)} d s\right)$. Therefore, the unconditional survival probability is

$$
\mathbb{P}^{\star}\left\{\tau_{i}>T\right\}=\mathbb{E}^{\star}\left\{e^{-\int_{0}^{T} X_{s}^{(i)}}\right\},
$$

with the expectation taken with respect to the risk neutral pricing measure $\mathbb{P}^{\star}$.

Consider a subset $\left\{i_{1}, \cdots, i_{n}\right\}$ of obligors. The probability of the joint survival of this set till time $T$ is then, under the doubly stochastic framework,

$$
\mathbb{P}^{\star}\left\{\tau_{i_{1}}>T, \cdots, \tau_{i_{n}}>T\right\}=\mathbb{E}^{\star}\left\{e^{-\sum_{j=1}^{n} \int_{0}^{T} X_{s}^{\left(i_{j}\right)} d s}\right\}
$$

\subsection{Vasicek Intensities and Survival Probabilities}

We start by considering $N$ names whose intensities $\left(X_{t}^{(i)}\right)$ are given by correlated Ornstein-Uhlenbeck processes $1 \leq i \leq N$. This corresponds to the Vasicek model

$$
d X_{t}^{(i)}=\kappa_{i}\left(\theta_{i}-X_{t}^{(i)}\right) d t+\sigma_{i} d W_{t}^{(i)}
$$

where the $\left(W_{t}^{(i)}\right)$ are correlated Brownian motions, with the correlation matrix $\mathbf{c}$ given by:

$$
d\left\langle W^{(i)}, W^{(j)}\right\rangle_{t}=c_{i j} d t
$$

We denote the survival probability for name $i$ by

$$
S_{i}\left(T ; x_{i}\right)=\mathbb{P}^{\star}\left(\tau_{i}>T \mid X_{0}^{(i)}=x_{i}\right)=\mathbb{E}^{\star}\left\{e^{-\int_{0}^{T} X_{s}^{(i)} d s} \mid X_{0}^{(i)}=x_{i}\right\} .
$$

We also denote the joint survival probability of all $N$ names by

$$
S(T ; \mathbf{x}, N)=\mathbb{E}^{\star}\left\{e^{-\int_{0}^{T} \sum_{i=1}^{N} X_{s}^{(i)} d s} \mid X_{0}^{(1)}=x_{1}, \cdots, X_{0}^{(N)}=x_{N}\right\},
$$

with $\mathbf{x}=\left(x_{1}, \cdots, x_{N}\right) \in \mathbb{R}^{N}$. From the Feynman-Kac formula it follows that the joint survival probability from time $t$ till time $T$

$$
u(t, \mathbf{x})=\mathbb{E}^{\star}\left\{e^{-\int_{t}^{T} \sum_{i=1}^{N} X_{s}^{(i)} d s} \mid \mathbf{X}_{t}=\mathbf{x}\right\},
$$

solves the partial differential equation

$$
\frac{\partial u}{\partial t}+\frac{1}{2} \sum_{i, j=1}^{N}\left(\sigma_{i} \sigma_{j} c_{i j}\right) \frac{\partial^{2} u}{\partial x_{i} \partial x_{j}}+\sum_{i=1}^{N} \kappa_{i}\left(\theta_{i}-x_{i}\right) \frac{\partial u}{\partial x_{i}}-\left(\sum_{i=1}^{N} x_{i}\right) u=0
$$

with terminal condition $u(T, \mathbf{x})=1$. 
Assume first that the covariance matrix $\mathbf{c}$ is the identity matrix, corresponding to the components of the intensity process $\mathbf{X}$ being independent. Then, as is well known, or can be readily checked, the solution is given by

$$
u(t, \mathbf{x})=\prod_{i=1}^{N} A_{i}(T-t) e^{-B_{i}(T-t) x_{i}},
$$

where we introduce

$$
\begin{aligned}
& B_{i}(s)=\int_{0}^{s} e^{-\kappa_{i} \xi} d \xi=\frac{1-e^{-\kappa_{i} \tau}}{\kappa_{i}}, \\
& A_{i}(s)=e^{-\theta_{i} \int_{0}^{s} \kappa_{i} B_{i}(\xi) d \xi+\frac{1}{2} \sigma_{i}^{2} \int_{0}^{s} B_{i}^{2}(\xi) d \xi}=e^{-\left(\theta_{i}^{d}\left(s-B_{i}(s)\right)+\frac{\sigma_{i}^{2}}{4 \kappa_{i}} B_{i}^{2}(s)\right)},
\end{aligned}
$$

and $\theta_{i}^{d}=\theta_{i}-\frac{\sigma_{i}^{2}}{2 \kappa_{i}^{2}}$.

In the general correlated case, we can write

$$
u(t, \mathbf{x})=A^{c}(T-t) \prod_{i=1}^{N} A_{i}(T-t) e^{-B_{i}(T-t) x_{i}},
$$

with

$$
A^{c}(s)=e^{\frac{1}{2} \sum_{i=1}^{N} \sum_{j \neq i=1}^{N}\left(\sigma_{i} \sigma_{j} c_{i j}\right) \int_{0}^{s} B_{i}(\xi) B_{j}(\xi) d \xi} .
$$

The last integral is given explicitly by

$$
\int_{0}^{s} B_{i}(\xi) B_{j}(\xi) d \xi=\frac{s}{\kappa_{i} \kappa_{j}}-\frac{B_{i}(s)}{\kappa_{i}\left(\kappa_{i}+\kappa_{j}\right)}-\frac{B_{j}(s)}{\kappa_{j}\left(\kappa_{i}+\kappa_{j}\right)}-\frac{B_{i}(s) B_{j}(s)}{\left(\kappa_{i}+\kappa_{j}\right)} .
$$

\section{Symmetric Name Case}

In this section, we analyze the symmetric names case where the dynamics and the starting points of the intensities are the same for all the names. This is convenient to understand the effects of the correlation and the size of the portfolio. We return to the heterogeneous case in Section 4.

Specifically, we have

$$
d X_{t}^{(i)}=\kappa\left(\theta-X_{t}^{(i)}\right) d t+\sigma d W_{t}^{(i)}, \quad X_{0}^{(i)}=x,
$$

with the parameters $\kappa, \theta \sigma$ and $x$ assumed constant and positive. Moreover, we assume that the correlation matrix is defined by $c_{i j}=\rho_{X}$, for $i \neq j$, with $\rho_{X} \geq 0$, and ones on the diagonal. We remark that such a correlation structure can be obtained by letting

$$
W_{t}^{(i)}=\sqrt{1-\rho_{X}} \tilde{W}_{t}^{(i)}+\sqrt{\rho_{X}} \tilde{W}_{t}^{(0)},
$$

where $\tilde{W}^{(i)}, i=0,1, \cdots, N$, are independent standard Brownian motions.

It follows from (6) that the joint survival probability for $n$ given names, say the first $n$ names, is

$$
\begin{aligned}
S(T ;(x, \cdots, x), n) & =\mathbb{E}^{\star}\left\{e^{-\int_{0}^{T}\left(X_{s}^{(1)}+\cdots+X_{s}^{(n)}\right) d s} \mid X_{0}^{(1)}=x, \cdots, X_{0}^{(n)}=x\right\} \\
& =e^{-n\left[\theta_{\infty}(T-B(T))+\left[1+(n-1) \rho_{X}\right] \sigma^{2} B^{2}(T) /(4 \kappa)+x B(T)\right]}
\end{aligned}
$$


with

$$
\begin{aligned}
B(T) & =\frac{1-e^{-\kappa T}}{\kappa}, \\
\theta_{\infty} & =\theta-\left[1+(n-1) \rho_{X}\right] \frac{\sigma^{2}}{2 \kappa^{2}} .
\end{aligned}
$$

This expression shows explicitly how the joint survival probability depends on the correlation $\rho_{X}$ and the "basket" size $n$. Note in particular how the basket size enhances the correlation effect. We consider next how a characterization of the loss distribution follows from (10).

\subsection{The Loss Distribution}

The loss distribution at time $T$ of a basket of size $N$ is given by its mass function

$$
p_{n}=\mathbb{P}^{\star}\{(\# \text { names defaulted at time } T)=n\}, \quad n=0,1, \cdots, N,
$$

and is explicitly

$$
p_{n}=\left(\begin{array}{c}
N \\
n
\end{array}\right) \sum_{j=0}^{n}\left(\begin{array}{c}
n \\
j
\end{array}\right) S_{N+j-n}(-1)^{j},
$$

using the short hand notation $S_{n}=S(T ;(x, \cdots, x), n)$ for the joint survival probability of $n$ names (for a derivation of this classical formula, see for instance [10]). This gives rise to an $\mathcal{O}\left(N^{2}\right)$ procedure for calculating the loss distribution.

However, a direct implementation of this formula is not numerically stable due to catastrophic cancellation errors in finite precision arithmetics. We comment therefore on an alternative implementation of (13). Note first that, from the formula (10) for the survival probability, we can write

$$
S_{n}=e^{-d_{1} n+d_{2} n^{2}}
$$

with $d_{i}$ explicitly given as

$$
\begin{aligned}
d_{1} & =d_{1}(T, x)=\theta T+(x-\theta) B(T)-\frac{1}{2} \sigma^{2}\left(1-\rho_{X}\right) B^{(2)}(T), \\
d_{2} & =d_{2}(T)=\frac{1}{2} \sigma^{2} \rho_{X} B^{(2)}(T), \\
B^{(2)}(T) & =\int_{0}^{T} B^{2}(s) d s=\frac{(T-B(T))}{\kappa^{2}}-\frac{B(T)^{2}}{2 \kappa},
\end{aligned}
$$

and we assume that the model parameters are chosen so that $d_{1}>0$. In the independent case $\rho_{X}=0$, we get the binomial distribution:

$$
p_{n}=\left(\begin{array}{c}
N \\
n
\end{array}\right)\left(1-e^{-d_{1}}\right)^{n} e^{-(N-n) d_{1}}=: \tilde{p}_{n}\left(d_{1}\right) .
$$

In the general case, we can write

$$
S_{n}=\mathbb{E}\left\{e^{-d_{1} n+n \sqrt{2 d_{2}} Z}\right\},
$$

for $Z$ a zero mean unit variance Gaussian random variable. Therefore, in the general case we find

$$
p_{n}=\mathbb{E}\left\{\tilde{p}_{n}\left(d_{1}+\sqrt{2 d_{2}} Z\right)\right\}
$$


Thus, we get the loss distribution stably and fast by integrating (non-negative) binomial distributions with respect to the Gaussian density. We remark that this essentially corresponds to conditioning with respect to the correlating Brownian motion, $W^{(0)}$, in (9). The argument $d_{1}+\sqrt{2 d_{2}} Z$ will be negative for $Z$ negative and with large magnitude. This reflects the fact that we are using a Vasicek model where the intensity may be negative. Below, we condition the Gaussian density to $Z>d_{1} / \sqrt{2 d_{2}}$ and choose parameters such that the complementary event has probability less than $10^{-3}$.

\subsection{Example with Constant Parameters and Strong Correlations}

In the model (1) we choose the parameters

$$
\theta=.02, \quad \kappa=.5, \quad \sigma=.015, \quad x=.02,
$$

and we let time to maturity $T=5$ and the number of names $N=125$, corresponding to th most common CDO contracts on the CDX and iTraxx. The loss distributions with $\rho_{X}=0$ and $\rho_{X}=.75$ respectively are shown in Figure 1 (left). Note how the strong correlation widens the loss distribution. Hence, it will strongly affect tranche prices. We consider the tranche prices for the CDX, defined in Section 2.1. The short rate is chosen to be fixed at $3 \%$, and the recovery is $40 \%$. In Figure 1 (right) we show the tranche prices plotted against the upper attachment point of each tranche, that are associated with the loss distributions on the left. The top plot is on a linear scale and the bottom on $\log$ scale to visually resolve well the senior tranches. Note how the strong correlations affects all tranches and that its relative effects are strongest for the senior tranches. The equity tranche is also strongly affected by the correlation with a negative correction.
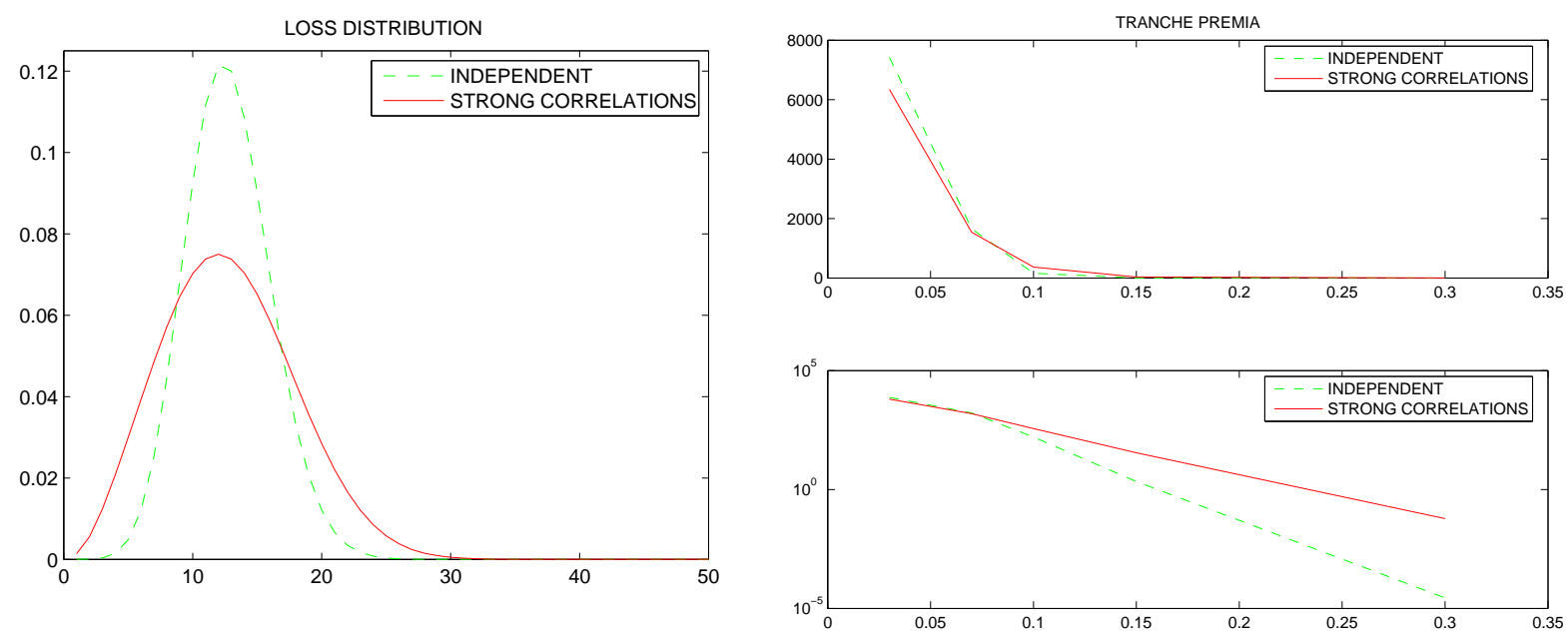

Figure 1: Loss distribution (left) and CDX tranche prices against upper attachment point (right), without and with strong correlations in between names.

\section{Stochastic Volatility Effects}

Stochastic volatility driven by common factors varying on fast and slow time scales has been shown to be effective in the structural approach, for capturing the yield spreads of single-name defaultable bonds [9], and multiname loss distributions [10]. Here we extend multiname intensity models, described in the previous section, to incorporate multiscale stochastic volatility. The intuitive idea is 
that simultaneous high volatility in intensity will generate clustering of defaults, giving the needed flexibility in the loss distribution. The slow factor gives additional freedom for the term structure.

Under the risk-neutral probability measure we assume the model

$$
d X_{t}^{(i)}=\kappa_{i}\left(\theta_{i}-X_{t}^{(i)}\right) d t+\sigma_{t}^{(i)} d W_{t}^{(i)},
$$

for $1 \leq i \leq N$ where the $W^{(j)}$ 's are correlated Brownian motions as in (2). The volatilities are stochastic and depend on a fast evolving factor $Y$ and slowly evolving factor $Z$ :

$$
\sigma_{t}^{(i)}=\sigma_{i}\left(Y_{t}, Z_{t}\right)
$$

where the functions $\sigma_{i}(y, z)$ are positive, bounded and bounded away from zero, and smooth in the second variable.

The fast process is modeled by

$$
d Y_{t}=\frac{1}{\varepsilon}\left(m-Y_{t}\right) d t+\frac{\nu \sqrt{2}}{\sqrt{\varepsilon}} d W_{t}^{(y)}
$$

with the small parameter $\varepsilon$ corresponding to the short time scale of the process $Y$. In fact, it is not important which particular model we choose for the fast scale, the important aspects of the process $Y$ are that it is ergodic and that it evolves on a fast time scale. We assume the correlations

$$
d\left\langle W^{(i)}, W^{(y)}\right\rangle_{t}=\rho_{Y} d t, \quad \text { for } \quad 1 \leq i \leq N .
$$

The slow factor evolves as

$$
d Z_{t}=\delta c(Z) d t+\sqrt{\delta} g(Z) d W_{t}^{(z)}
$$

with the large parameter $1 / \delta$ corresponding to the long time scale of the process $Z$.

The functions and $c$ and $g$ are assumed to be smooth and we assume the correlations

$$
d\left\langle W^{(i)}, W^{(z)}\right\rangle_{t}=\rho_{Z} d t
$$

We denote by $\rho_{Y Z}$ the correlation coefficient defined by

$$
d\left\langle W^{(y)}, W^{(z)}\right\rangle_{t}=\rho_{Y Z} d t
$$

We assume the coefficients $\rho_{Y}, \rho_{Z}, \rho_{Y Z}$ and the matrix $\left(c_{i j}\right)$ are such that the joint covariance matrix of the Brownian motions $W^{(i)}(i=1, \cdots, n), W^{(y)}$ and $W^{(z)}$ is non-negative definite.

The joint survival probabilities become now

$$
S(T ; \mathbf{x}, y, z, N)=\mathbb{E}^{\star}\left\{e^{-\sum_{i=1}^{N} \int_{0}^{T} X_{s}^{(i)} d s} \mid \mathbf{X}_{0}=\mathbf{x}, Y_{0}=y, Z_{0}=z\right\}
$$

In this case the joint survival probability from time $t$

$$
u^{\varepsilon, \delta}(t, \mathbf{x} ; y, z, N)=\mathbb{E}^{\star}\left\{e^{-\int_{t}^{T} \sum_{i=1}^{N} X_{s}^{(i)} d s} \mid \mathbf{X}_{t}=\mathbf{x}, Y_{t}=y, Z_{t}=z\right\}
$$

solves the partial differential equation

$$
\begin{aligned}
& \mathcal{L}^{\varepsilon, \delta} u^{\varepsilon, \delta}=0 \\
& u^{\varepsilon, \delta}(T, \mathbf{x}, y, z)=1
\end{aligned}
$$


with the notation

$$
\mathcal{L}^{\varepsilon, \delta}=\frac{\partial}{\partial t}+\mathcal{L}_{(\mathbf{x}, y, z)}-\left(\sum_{i=1}^{N} x_{i}\right) \cdot
$$

where $\mathcal{L}_{(\mathbf{x}, y, z)}$ denotes the infinitesimal generator of the Markov process $\left(\mathbf{X}_{t}, Y_{t}, Z_{t}\right)$ under the riskneutral measure.

We write the operator $\mathcal{L}^{\varepsilon, \delta}$ in terms of the small parameters $(\varepsilon, \delta)$ as

$$
\mathcal{L}^{\varepsilon, \delta}=\frac{1}{\varepsilon} \mathcal{L}_{0}+\frac{1}{\sqrt{\varepsilon}} \mathcal{L}_{1}+\mathcal{L}_{2}+\sqrt{\delta} \mathcal{M}_{1}+\delta \mathcal{M}_{2}+\sqrt{\frac{\delta}{\varepsilon}} \mathcal{M}_{3}
$$

where the operators $\mathcal{L}_{k}$ and $\mathcal{M}_{k}$ are defined by:

$$
\begin{aligned}
\mathcal{L}_{0} & =\nu^{2} \frac{\partial^{2}}{\partial y^{2}}+(m-y) \frac{\partial}{\partial y} \\
\mathcal{L}_{1} & =\sqrt{2} \nu \rho_{Y} \sum_{i=1}^{N} \sigma_{i}(y, z) \frac{\partial^{2}}{\partial x_{i} \partial y} \\
\mathcal{L}_{2} & =\frac{\partial}{\partial t}+\frac{1}{2} \sum_{i, j=1}^{N} c_{i j} \sigma_{i}(y, z) \sigma_{j}(y, z) \frac{\partial^{2}}{\partial x_{i} \partial x_{j}}+\sum_{i=1}^{N} \kappa_{i}\left(\theta_{i}-x_{i}\right) \frac{\partial}{\partial x_{i}}-\left(\sum_{i=1}^{N} x_{i}\right) \\
\mathcal{M}_{1} & =\rho_{Z} g(z) \sum_{i=1}^{N} \sigma_{i}(y, z) \frac{\partial^{2}}{\partial x_{i} \partial z} \\
\mathcal{M}_{2} & =\frac{1}{2} g^{2}(z) \frac{\partial^{2}}{\partial z^{2}}+c(z) \frac{\partial}{\partial z} \\
\mathcal{M}_{3} & =\sqrt{2} \nu g(z) \rho_{Y Z} \frac{\partial^{2}}{\partial y \partial z}
\end{aligned}
$$

Note that

- $\varepsilon^{-1} \mathcal{L}_{0}$ is the infinitesimal generator of the Ornstein Uhlenbeck process $Y$,

- $\mathcal{L}_{1}$ contains the mixed derivatives due to the correlation between $\mathbf{X}$ and $Y$,

- $\mathcal{L}_{2}$ is the differential operator corresponding to the unperturbed problem in (3), but evaluated at the volatilities $\sigma_{i}(y, z)$,

- $\mathcal{M}_{1}$ contains the mixed derivatives due to the correlation between $\mathbf{X}$ and $Z$,

- $\delta \mathcal{M}_{2}$ is the infinitesimal generator of the process $Z$,

- $\mathcal{M}_{3}$ contains the mixed derivatives due to the correlation between $Y$ and $Z$.

We will next present the results of the singular and regular perturbation techniques framework introduced in [8] and adapted to credit risk to obtain an accurate characterization of the loss distribution in the regime where $\varepsilon$ and $\delta$ are small. This will enable us to describe how the fluctuations in the volatility affect the loss distribution and tranche prices. 


\subsection{Time-Scale Perturbations}

We expand the survival probability $u^{\varepsilon, \delta}$ defined in (19), in the small parameters $\varepsilon$ and $\delta$ as

$$
u^{\varepsilon, \delta} \sim \tilde{u}^{\varepsilon, \delta}=u_{0}+\sqrt{\varepsilon} u_{1,0}+\sqrt{\delta} u_{0,1},
$$

where $u_{1,0}$ and $u_{0,1}$ are the first corrections due to fast and slow volatility scales respectively.

The leading order term $u_{0}$ of the joint survival probability is obtained by solving the problem (3) with the effective diffusion matrix

$$
[\mathbf{d}(z)]_{i j}=d_{i j}(z):=\int c_{i j} \sigma_{i}(y, z) \sigma_{j}(y, z) \Phi(y) d y
$$

with $\Phi$ being the invariant distribution for the $Y$ process. Since the process $Y$ evolves on the fast scale its leading order effect is obtained by integration with respect to $\Phi$. The process $Z$ evolves on a relatively slow scale and at this level of approximation its effect corresponds to just evaluating this process at its current "frozen" level $z$. We introduce the effective operator

$$
\mathcal{L}^{e}(\mathbf{d}(z))=\frac{\partial}{\partial t}+\frac{1}{2} \sum_{i, j=1}^{N} d_{i j}(z) \frac{\partial^{2}}{\partial x_{i} \partial x_{j}}+\sum_{i=1}^{N} \kappa_{i}\left(\theta_{i}-x_{i}\right) \frac{\partial}{\partial x_{i}}-\left(\sum_{i=1}^{N} x_{i}\right)
$$

then we have

Definition 1 The leading order term $u_{0}$ is the survival probability which solves

$$
\mathcal{L}^{e}(\mathbf{d}(\mathbf{z})) u_{0}=0, \quad u_{0}(T, \mathbf{x} ; z)=1 .
$$

As in the constant volatility case of Section 2.3, the solution is given by

$$
u_{0}(t, \mathbf{x} ; z)=A^{c}(T-t) \prod_{i=1}^{N} A_{i}(T-t) e^{-B_{i}(T-t) x_{i}},
$$

with $B_{i}$ defined in (4), and

$$
A^{c}(s)=e^{\frac{1}{2} \sum_{i=1}^{N} \sum_{j \neq i=1}^{N}\left(d_{i j}(z)\right) \int_{0}^{s} B_{i}(\xi) B_{j}(\xi) d \xi} .
$$

The last integral is given explicitly in (8).

Next, we obtain $u_{1,0}$, the correction to the survival probability due to the fast volatility factor $Y$. We start by introducing the operator

$$
\mathcal{A}_{1,0}=\left\langle\mathcal{L}_{1} \mathcal{L}_{0}^{-1}\left(\mathcal{L}_{2}-\left\langle\mathcal{L}_{2}\right\rangle\right)\right\rangle,
$$

which will be given explicitly below and where the triangular brackets represent integration with respect to the invariant distribution for the $Y$ process.

Definition 2 The function $u_{1,0}(t, \mathbf{x}, z)$ solves the inhomogeneous problem

$$
\begin{aligned}
\mathcal{L}^{e}(\mathbf{d}(z)) u_{1,0} & =\mathcal{A}_{1,0} u_{0} \\
u_{1,0}(T, \mathbf{x} ; z) & =0 .
\end{aligned}
$$


Thus, $u_{1,0}$ solves a linear equation with the effective operator $\mathcal{L}^{e}(\mathbf{d}(z))$, but now the problem involves a source term $\mathcal{A}_{1,0} u_{0}$, defined in terms of the leading order survival probability $u_{0}$, and with a zero terminal condition.

We consider next the correction $u_{0,1}$ due to the slow volatility factor. In this case we introduce the operator

$$
\mathcal{A}_{0,1}=-\left\langle\mathcal{M}_{1}\right\rangle
$$

and obtain:

Definition 3 The function $u_{0,1}(t, \mathbf{x}, z)$ is the solution of the problem

$$
\begin{aligned}
& \mathcal{L}^{e}(\mathbf{d}(z)) u_{0,1}=\mathcal{A}_{0,1} u_{0}, \\
& u_{0,1}(T, \mathbf{x} ; z)=0
\end{aligned}
$$

which is again a source problem with respect to the operator $\mathcal{L}^{e}(\mathbf{d}(z))$ and with a zero terminal condition.

The PDE problems for the approximation terms given in Definitions 1, 2 and 3 can be motivated by formal multiscale asymptotics. Similar calculations in the case of equity stochastic volatility models appear in [8], and the formal asymptotics are identical albeit with different definitions of the operators $\mathcal{L}_{k}$ and $\mathcal{M}_{k}$. In the present case, $\mathcal{L}_{2}$ is associated with a multi-dimensional diffusion process, which does not present major difficulties compared with the single-dimensional equity case in [8]. The unboundedness of the $X^{(i)}$ raises some technical issues, which were addressed in the one-dimensional case in [1]. A precise accuracy result is given at the end of this section.

We next obtain an expression for $\tilde{u}^{\varepsilon, \delta}$ and start by introducing the symmetric matrix $\Psi(y, z)$ satisfying

$$
\mathcal{L}_{0} \Psi_{i_{1}, i_{2}}=c_{i_{1}, i_{2}} \sigma_{i_{1}}(y, z) \sigma_{i_{2}}(y, z)-d_{i_{1}, i_{2}}(z)
$$

and the coefficients

$$
V_{3}^{\varepsilon}\left(z, i_{1}, i_{2}, i_{3}\right)=-\sqrt{\varepsilon} \frac{\rho_{Y} \nu}{\sqrt{2}}\left\langle\sigma_{i_{3}} \frac{\partial \Psi_{i_{1} i_{2}}}{\partial y}\right\rangle .
$$

Using the definitions in $(21)$ - $(23)$ one then obtains that the scaled operator $\sqrt{\varepsilon} \mathcal{A}_{1,0}$ can be written

$$
\sqrt{\varepsilon} \mathcal{A}_{1,0}=-\sum_{i_{1}, i_{2}, i_{3}=1}^{N} V_{3}^{\varepsilon}\left(z, i_{1}, i_{2}, i_{3}\right) \frac{\partial^{3}}{\partial x_{i_{1}} \partial x_{i_{2}} \partial x_{i_{3}}} .
$$

We make the ansatz $\sqrt{\varepsilon} u_{1,0}(t, \mathbf{x} ; z)=D(T-t) u_{0}(t, \mathbf{x} ; z)$, which leads to the ODE for D:

$$
-D^{\prime}=\sum_{i_{1}, i_{2}, i_{3}=1}^{N} V_{3}^{\varepsilon}\left(z, i_{1}, i_{2}, i_{3}\right) B_{i_{1}} B_{i_{2}} B_{i_{3}}, \quad D(0)=0,
$$

using the expressions (5) and (7) for the survival probability in the constant volatility case. Therefore, we compute the form for the correction due to fast volatility fluctuations

$$
\sqrt{\varepsilon} u_{1,0}=\left(\sum_{i_{1}, i_{2}, i_{3}=1}^{N} V_{3}^{\varepsilon}\left(z, i_{1}, i_{2}, i_{3}\right) \int_{0}^{T} B_{i_{1}}(s) B_{i_{2}}(s) B_{i_{3}}(s) d s\right) u_{0} .
$$

Thus, the correction depends on the underlying model structure in a complicated way, but only the effective market group parameters $V_{3}^{\varepsilon}(\cdot)$ are needed to compute the fast time scale correction $u_{1,0}$. 
Using next the definition (24) we find that the (scaled) operator $\sqrt{\delta} \mathcal{A}_{0,1}$ in the source term of the $u_{0,1}$ problem $(27)$ can be written

$$
\sqrt{\delta} \mathcal{A}_{0,1}=-\sqrt{\delta}\left\langle\mathcal{M}_{1}\right\rangle=-\sum_{i=1}^{N} V_{1}^{\delta}(z, i) \frac{\partial^{2}}{\partial z \partial x_{i}}
$$

where we introduced the coefficients

$$
V_{1}^{\delta}(z, i)=\sqrt{\delta} g(z) \rho_{Z}\left\langle\sigma_{i}\right\rangle
$$

It then follows, again using the expressions (5) and (7), that

$$
\sqrt{\delta} u_{0,1}=\left(\frac{1}{2} \sum_{i_{1}=1}^{N} V_{1}^{\delta}\left(z, i_{1}\right) \sum_{i_{2}=1}^{N} \sum_{i_{3}=1}^{N} \frac{\mathrm{d}}{\mathrm{d} z}\left(d_{i_{1} i_{2}}\right) \int_{0}^{T} B_{i_{1}}(v) \int_{0}^{v} B_{i_{1}}(s) B_{i_{2}}(s) d s d v\right) u_{0} .
$$

Note that the effective market parameters $V_{3}^{\varepsilon}$ and $V_{1}^{\delta}$ depend on the underlying model in a complicated way as explained above. However, this particular dependence will not be needed in applying the asymptotic theory since these market group parameters rather than the full underlying model will be calibrated to market data.

We end this section with an accuracy result for our approximation (25).

Theorem 4.1 For any fixed $t<T, \mathbf{x} \in \mathbb{R}^{N}$ and $y, z \in \mathbb{R}$,

$$
\left|u^{\varepsilon, \delta}(t, \mathbf{x}, y, z)-\left(u_{0}(t, \mathbf{x}, z)+\sqrt{\varepsilon} u_{1,0}(t, \mathbf{x}, z)+\sqrt{\delta} u_{0,1}(t, \mathbf{x}, z)\right)\right|=\mathcal{O}(\varepsilon+\delta),
$$

where $u^{\varepsilon, \delta}$ is the solution of the original problem (20), and $u_{0}, \sqrt{\varepsilon} u_{1,0}$ and $\sqrt{\delta} u_{0,1}$ are given by (26), (28) and (29) respectively.

Sketch of Proof: One of the difficulties is that the potential $\left(-\sum_{i} x_{i}\right)$ in $(20)$ is unbounded from above since the processes $X^{(i)}$ are unbounded from below. As in [1] (in the one-dimensional case), the transformation

$$
u^{\varepsilon, \delta}(t, \mathbf{x}, y, z)=M^{\varepsilon, \delta}(t, y, z) \prod_{i=1}^{N} e^{-B_{i}(T-t) x_{i}}
$$

reduces to a Feynman-Kac equation for $M$ with a bounded potential in $(y, z)$, bounded timedependent coefficients, and smooth terminal condition $M^{\varepsilon, \delta}(T, y, z)=1$. The rest of the proof follows readily from the proof of accuracy given in [7, Chapter 5] with the fast factor, and generalized to fast and slow factors in [8].

\subsection{Stochastic Volatility Effects in the Symmetric Case}

We consider the simplified form for the asymptotic survival probabilities in the symmetric case considered in Section 4.2, with stochastic volatilties $\sigma_{t}^{(i)} \equiv \sigma\left(Y_{t}, Z_{t}\right)$. One readily computes that in this case

$$
\begin{aligned}
& S(T ;(x, \cdots, x), y, z, n)=u^{\varepsilon, \delta}(0 ;(x, \cdots, x), y, z, n) \sim \tilde{u}^{\varepsilon, \delta}(0 ;(x, \cdots, x), z, n) \\
& \quad=\left(1+D^{\varepsilon}(T ; z, n)+D^{\delta}(T ; z, n)\right) e^{-n\left[\theta_{\infty}(z)(T-B(T))+\left[1+(n-1) \rho_{X}\right] \bar{\sigma}^{2}(z) B^{2}(T) /(4 \kappa)+x B(T)\right]}
\end{aligned}
$$


where

$$
\begin{aligned}
\bar{\sigma}^{2}(z) & =\left\langle\sigma(\cdot, z)^{2}\right\rangle \\
D^{\varepsilon}(T ; z, n) & =v_{3}(z) n^{2}\left(1+(n-1) \rho_{X}\right) B^{(3)}(T), \\
D^{\delta}(T ; z, n) & =v_{1}(z) n^{2}\left(1+(n-1) \rho_{X}\right) \tilde{B}^{(3)}(T), \\
B^{(3)}(T) & =\int_{0}^{T} B^{3}(s) d s, \\
\tilde{B}^{(3)}(T) & =\int_{0}^{T} B(s) B^{(2)}(s) d s \\
v_{1}(z) & =\frac{\sqrt{\delta}}{2} g(z) \rho_{Z}\langle\sigma(\cdot, z)\rangle \frac{\partial}{\partial z}\left\langle\sigma^{2}(\cdot, z)\right\rangle, \\
v_{3}(z) & =-\sqrt{\varepsilon} \frac{\rho_{Y} \nu}{\sqrt{2}}\left\langle\sigma(\cdot, z) \frac{\partial \Psi(\cdot, z)}{\partial y}\right\rangle,
\end{aligned}
$$

with here $\Psi$ being a solution to the Poisson equation in the $y$-variable:

$$
\mathcal{L}_{0} \Psi=\sigma^{2}(y, z)-\left\langle\sigma^{2}(\cdot, z)\right\rangle .
$$

Note that $\theta_{\infty}$ is computed as in (11), but evaluated with $\sigma=\bar{\sigma}(z)$ :

$$
\theta_{\infty}(z)=\theta-\left[1+(n-1) \rho_{X}\right] \frac{\bar{\sigma}^{2}(z)}{2 \kappa^{2}}
$$

Therefore, using the notation introduced in (14), we have

$$
S(T ; x, z, n) \sim\left(1+n^{3} \rho_{X}\left(v_{3}(z) B^{(3)}(T)+v_{1}(z) \tilde{B}^{(3)}(T)\right)\right) e^{-n d_{1}(T, x, z)+n^{2} \tilde{d}_{2}(T, z)},
$$

with

$$
\tilde{d}_{2}(T, x, z)=d_{2}(T, x, z)+\left(1-\rho_{X}\right)\left(v_{3}(z) B^{(3)}(T)+v_{1}(z) \tilde{B}^{(3)}(T)\right),
$$

where $d_{1}$ and $d_{2}$ are computed as in (15) and (16):

$$
\begin{aligned}
d_{1}(T, x, z) & =\theta T+(x-\theta) B(T)-\frac{1}{2}\left(1-\rho_{X}\right) \bar{\sigma}(z)^{2} B^{(2)}(T), \\
d_{2}(T, z) & =\frac{1}{2} \rho_{X} \bar{\sigma}(z)^{2} B^{(2)}(T), \\
B^{(2)}(T) & =\int_{0}^{T} B^{2}(s) d s .
\end{aligned}
$$

By the remarks following equation (14), we easily compute the loss distribution that follows from (34) in the case $\rho_{X}=0$. That is, we compute it by (17) with

$$
\begin{aligned}
\tilde{p}_{n}\left(x^{\prime}\right)= & \left(\begin{array}{c}
N \\
n
\end{array}\right) \sum_{j=0}^{n}\left(\begin{array}{c}
n \\
j
\end{array}\right) e^{-x^{\prime}(N+j-n)}(-1)^{j} \\
= & \left(\begin{array}{c}
N \\
n
\end{array}\right)\left(1-e^{-x^{\prime}}\right)^{n} e^{-(N-n) x^{\prime}},
\end{aligned}
$$


and $d_{2}$ replaced by $\tilde{d}_{2}$. In the general case with $\rho_{X} \neq 0$ we obtain the loss distribution by the generalization of (17):

$$
p_{n}=\mathbb{E}\left\{\tilde{p}_{n}\left(d_{1}+\sqrt{2 \tilde{d}_{2}} Z\right)+\left(\rho_{X}\left(v_{3} B^{(3)}+v_{1} \tilde{B}^{(3)}\right)\right) \tilde{p}_{n}^{\prime \prime \prime}\left(d_{1}+\sqrt{2 \tilde{d}_{2}} Z\right)\right\},
$$

where $Z$ is an $\mathcal{N}(0,1)$ random variable. Note that

$$
\sum_{n=0}^{N} \tilde{p}_{n}^{\prime \prime \prime}(x)=\frac{d^{3}}{d x^{3}} \sum_{n=0}^{N} \tilde{p}_{n}(x)=0,
$$

so that indeed $\sum_{n=0}^{N} p_{n}=1$. We remark however that outside of the domain of validity of the approximation we may have $p_{n}<0$. Thus, when applying the approximation the $v_{i}$ 's must be chosen small enough so that indeed the computed $p_{n}$ 's define a distribution. In the modeling above the $v_{i}$ 's are $\mathcal{O}(\sqrt{\delta}, \sqrt{\varepsilon})$ and are therefore small. From the representation (38) we see that the effect of the stochastic volatility in the uncorrelated case with $\rho_{X}=0$ is a modification of the hazard rate, to the order we consider. While the combined effect of correlation and stochastic volatility is qualitatively different and gives a correction to the binomial shape. We can also observe that the effects of the slow and fast volatility scales are qualitatively similar, giving the computed correction a canonical character, it gives the structure of the correction under a large class of underlying models. This will be further reinforced by our analysis below.

We next continue the numerical example introduced in Section 3.2. We choose the parameters as

$$
\theta=.03, \quad \kappa=.5, \quad \sigma=.02, \quad x=.03,
$$

and we let the time to maturity $T=5$ and the number of names $N=125$. The short rate is chosen to be fixed at $3 \%$ and the recovery is $40 \%$ as before. Here and below, when we show numerical examples they are based on the asymptotic approximations of the type (38). Our analysis has shown that name correlation can be generated in various ways. Either by directly correlating the innovations or Brownian motions driving the hazard rates of the names or alternatively by introducing time scale effects in the volatility. We remark though that the time evolution of the loss distribution depends somewhat on how the correlation is generated. In Figure 2, we illustrate the relative strong effect that combined name correlation and stochastic volatility has, here with $\rho_{X}=.01$ and $v_{3}=3 \cdot 10^{-4}$. We let $v_{1}=0$, as the influence of this parameter is similar to that of $v_{3}$. Note in particular the relative strong effect on the senior tranches and that in this case the shape of the loss distribution is affected, however, the equity tranches are relatively less affected.

\subsection{Name Correlation via Fluctuations in Hazard Rate Level}

In the modeling above we assumed that the mean reversion level of the name hazard rates, the $\theta$ parameter, was constant. We next examine the effects of time variations in this parameter by generalizing the model in (18) as

$$
d X_{t}^{(i)}=\kappa\left(\theta\left(Y_{t}^{(2)}\right)-X_{t}^{(i)}\right) d t+\sigma\left(Y_{t}^{(1)}\right) d W_{t}^{(i)},
$$

for $1 \leq i \leq N$ where the $W^{(j)}$ 's are correlated Brownian motions as in (2). Note that here we consider only the case with a symmetric model and fast scale time fluctuations in the parameters. The general case can be analyzed with a similar approach. The fast processes are modeled by

$$
d Y_{t}^{(j)}=\frac{1}{\varepsilon}\left(m_{j}-Y_{t}^{(j)}\right) d t+\frac{\nu_{j} \sqrt{2}}{\sqrt{\varepsilon}} d W_{t}^{(j, y)}, \quad \text { for } \quad j \in\{1,2\} .
$$



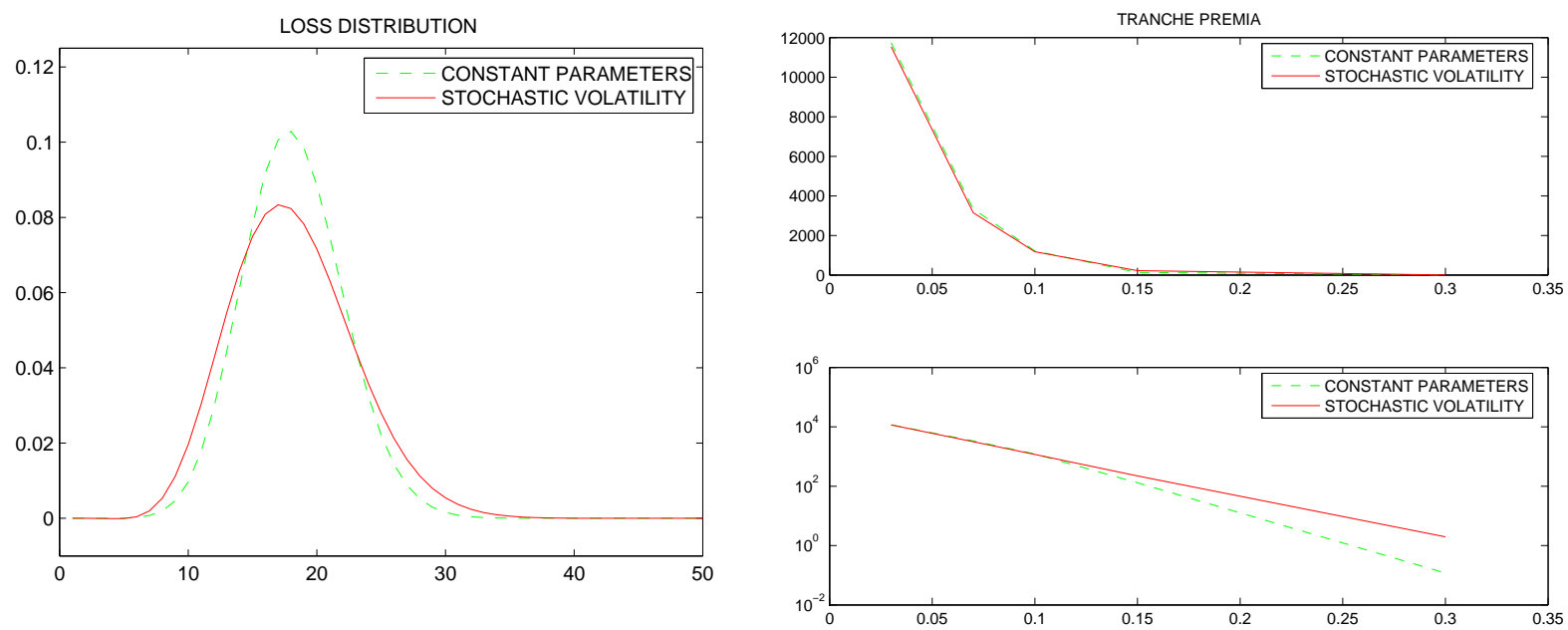

Figure 2: Loss distribution (left) and tranche prices (right) without and with strong stochastic volatility.

and we assume the correlations

$$
d\left\langle W^{(i)}, W^{(j, y)}\right\rangle_{t}=\rho_{Y_{j}} d t, \quad \text { for } \quad 1 \leq i \leq N \quad \text { and } \quad j \in\{1,2\},
$$

which can be decomposed in terms of independent Brownian motions $\left(\tilde{W}_{t}^{(0)}, \tilde{W}_{t}^{(j, y)}\right)$ as follows:

$$
\begin{aligned}
W_{t}^{(j, y)} & =\sqrt{1-\rho_{y_{j}}} \tilde{W}_{t}^{(j, y)}+\sqrt{\rho_{y_{j}}} \tilde{W}_{t}^{(0)}, \\
\rho_{y_{j}} & =\rho_{Y_{j}} / \sqrt{\rho_{X}} .
\end{aligned}
$$

Thus, we assume that there is one short time scale associated with the market, the time scale characterized by $\varepsilon$.

In (25) we now only have the correction due to the fast scale variation

$$
u^{\varepsilon} \sim \tilde{u}^{\varepsilon}=u_{0}+\sqrt{\varepsilon} u_{1,0} .
$$

The functions $u_{0}$ and $u_{1,0}$ solves again the problems in Definition 1 and Definition 2 respectively, upon the replacements

$$
\begin{aligned}
\mathcal{L}_{0} & \mapsto \sum_{j=1}^{2}\left(\nu_{j}^{2} \frac{\partial^{2}}{\partial y_{j}^{2}}+\left(m_{j}-y_{j}\right) \frac{\partial}{\partial y_{j}}\right)+\sqrt{\rho_{y_{1}} \rho_{y_{2}}} \nu_{1} \nu_{2} \frac{\partial^{2}}{\partial y_{1} \partial y_{2}} \\
\mathcal{L}_{1} & \mapsto \sum_{j=1}^{2} \sqrt{2} \nu_{j} \rho_{Y_{j}} \sigma\left(y_{1}\right) \sum_{i=1}^{N} \frac{\partial^{2}}{\partial x_{i} \partial y_{j}}, \\
\mathcal{L}_{2} & \mapsto \frac{\partial}{\partial t}+\frac{\sigma^{2}\left(y_{1}\right)}{2} \sum_{i, j=1}^{N} c_{i j} \frac{\partial^{2}}{\partial x_{i} \partial x_{j}}+\sum_{i=1}^{N} \kappa\left(\theta\left(y_{2}\right)-x_{i}\right) \frac{\partial}{\partial x_{i}}-\left(\sum_{i=1}^{N} x_{i}\right) .
\end{aligned}
$$

It then follows that the expression (30) for the asymptotic loss distribution becomes:

$$
\begin{aligned}
& S(T ; x, n)=u^{\varepsilon}\left(0 ;(x, \cdots, x), y_{1}, y_{2}, n\right) \sim \tilde{u}^{\varepsilon}(0 ; x, n) \\
& =\left(1+D^{\varepsilon}(T ; n)+D_{\theta}^{\varepsilon}(T ; n)\right) e^{-n\left[\bar{\theta}_{\infty}(T-B(T))+\left[1+(n-1) \rho_{X}\right] \bar{\sigma}^{2} B^{2}(T) /(4 \kappa)+x B(T)\right]},
\end{aligned}
$$


with now

$$
\begin{aligned}
\bar{\sigma}^{2} & =\left\langle\sigma^{2}(\cdot)\right\rangle_{1} \\
\bar{\theta} & =\langle\theta(\cdot)\rangle_{2} \\
\bar{\theta}_{\infty}(z) & =\bar{\theta}-\left[1+(n-1) \rho_{X}\right] \frac{\bar{\sigma}^{2}}{2 \kappa^{2}},
\end{aligned}
$$

where $\langle\cdot\rangle_{i}$ denotes integration with respect to the invariant distribution for $Y^{(i)}, i \in\{1,2\}$. Moreover, $D^{\varepsilon}$ is defined as in (32), but without the $z$ dependence:

$$
\begin{aligned}
D^{\varepsilon}(T ; n) & =v_{3} n^{2}\left(1+(n-1) \rho_{X}\right) B^{(3)}(T), \\
v_{3} & =-\sqrt{\varepsilon} \frac{\rho_{Y} \nu_{1}}{\sqrt{2}}\left\langle\sigma(\cdot) \frac{\partial \Psi(\cdot)}{\partial y}\right\rangle_{1}, \\
\mathcal{L}_{0,1} \Psi & =\sigma^{2}(y)-\left\langle\sigma^{2}(\cdot)\right\rangle_{1}
\end{aligned}
$$

with $\mathcal{L}_{0,1}$ being the infinitesimal generator for $Y_{1}$. The correction, $D_{\theta}^{\varepsilon}$, due to fluctuations in the parameter $\theta$ is given by:

$$
\begin{aligned}
D_{\theta}^{\varepsilon}(T ; n) & =v_{2} n^{2} B^{(2)}(T) \\
v_{2} & =-\sqrt{2 \varepsilon} \rho_{Y_{2}} \kappa \nu_{2}\left\langle\sigma\left(y_{1}\right) \frac{\partial \Psi_{2}\left(y_{2}\right)}{\partial y}\right\rangle_{1,2} \\
\mathcal{L}_{0,2} \Psi_{2} & =\theta(y)-\langle\theta(\cdot)\rangle_{2}
\end{aligned}
$$

with $\mathcal{L}_{0,2}$ being the infinitesimal generator for $Y^{(2)}$ and $\langle\cdot\rangle_{1,2}$ denoting integration with respect to the joint invariant distribution for $Y^{(1)}$ and $Y^{(2)}$.

Observe that the form of the joint survival probability is as in (30), which confirms the canonical structure of the correction to the survival probability due to multiscale parameter fluctuations. It also follows that we can use the same procedure as the one described in Section 4.2 for computing the loss distribution and associated tranche prices. In the present case we can write

$$
\begin{aligned}
& S(T ; x, n) \sim \tilde{u}^{\varepsilon}(0 ; x, n) \\
& =\left(1+c_{1} n^{2} B^{(2)}(T)+\left(c_{2} n^{2}+c_{3} n^{3}\right) B^{(3)}(T)\right) S_{0}(T ; x, n), \\
& S_{0}(T ; x, n)=e^{-n\left[\bar{\theta}_{\infty}(T-B(T))+\left[1+(n-1) \rho_{X}\right] \bar{\sigma}^{2} B^{2}(T) /(4 \kappa)+x B(T)\right]},
\end{aligned}
$$

with $c_{2}$ and $c_{3}$ calibration parameters of magnitude $\mathcal{O}(\sqrt{\varepsilon})$. Note that the form of the correction in (30) is identical with respect to the form in $n$, but slightly different in the temporal dependence. We continue in the next section by briefly discussing the case with a stochastic short rate and show that then we also get corrections terms that are $\mathcal{O}(1)$ and $\mathcal{O}(n)$.

\subsection{Short Rate Term Structure Effects}

Consider the case were the short rate is stochastic, also modeled as a Vasicek process under the risk neutral measure:

$$
\begin{aligned}
d X_{t}^{(i)} & =\kappa\left(\theta\left(Y^{(2)}\right)-X_{t}^{(i)}\right) d t+\sigma\left(Y^{(1)}\right) d W_{t}^{(i)} \\
d r_{t} & =\kappa\left(\theta_{r}\left(Y^{(4)}\right)-r_{t}\right) d t+\sigma_{r}\left(Y^{(3)}\right) d W_{t}^{r}
\end{aligned}
$$


We again assume a symmetric model with the time scales defined by $1 / \kappa$ and $1 / \varepsilon$ so that the fast scales are all modeled by:

$$
d Y_{t}^{(j)}=\frac{1}{\varepsilon}\left(m_{j}-Y_{t}^{(j)}\right) d t+\frac{\nu_{j} \sqrt{2}}{\sqrt{\varepsilon}} d W_{t}^{(j, y)}
$$

with the symmetric correlations of the same form as above:

$$
\begin{aligned}
d\left\langle W^{(i)}, W^{(j, y)}\right\rangle_{t} & =\rho_{Y_{j}} d t, \quad \text { for } \quad 1 \leq i \leq N, 1 \leq j \leq 4, \\
d\left\langle W^{r}, W^{(j, y)}\right\rangle_{t} & =\rho_{r, Y_{j}} d t, \quad \text { for } \quad 1 \leq j \leq 4, \\
d\left\langle W^{(i)}, W^{(j)}\right\rangle_{t} & =\rho_{X} d t, \quad \text { for } \quad i \neq j, \quad d\left\langle W^{(i)}, W^{r}\right\rangle_{t}=\rho_{r} d t, \quad \text { for } \quad 1 \leq i \leq N .
\end{aligned}
$$

In this case, the quantity of interest that we need to compute in order to price the CDO is:

$$
q^{(r)}(T ; x, n)=\mathbb{E}^{\star}\left\{e^{-\int_{0}^{T}\left(r(s)+X_{s}^{(1)}+\cdots+X_{s}^{(n)}\right) d s} \mid X_{0}^{(1)}=x, \cdots, X_{0}^{(n)}=x, r(0)=r_{0}\right\}
$$

the expectation under the risk neutral measure of the discounted joint survival probabilities. This expression is of the same form as in (6). By generalization of the multiscale analysis we then easily find the asymptotic approximation

$$
\begin{aligned}
& q^{(r)}(T ; x, n) \sim\left(1+\left(c_{0}+c_{1} n+c_{2} n^{2}+c_{3} n^{3}\right) B^{(3)}(T)\right) S_{0}^{(r)}(T ; x, n), \\
& S_{0}^{(r)}(T ; x, n)=e^{-n\left[\bar{\theta}_{\infty}(T-B(T))+\left[1+(n-1) \rho_{X}\right] \bar{\sigma}^{2} B^{2}(T) /(4 \kappa)+x B(T)\right]} \\
& \quad \times e^{-\left[\bar{\theta}_{r, \infty}(T-B(T))+\left[1+n \rho_{r}\right] \bar{\sigma}_{r}^{2} B^{2}(T) /(4 \kappa)+r_{0} B(T)\right]}
\end{aligned}
$$

with $c_{i}, i \in\{0, \cdots, 3\}$ calibration parameters of magnitude $\mathcal{O}(\sqrt{\varepsilon})$ and

$$
\begin{aligned}
\bar{\sigma}_{r}^{2} & =\left\langle\sigma_{r}^{2}(\cdot)\right\rangle_{3}, \\
\bar{\theta}_{r, \infty} & =\left\langle\theta_{r}(\cdot)\right\rangle_{4}-\left[1+n \rho_{r}\right] \frac{\bar{\sigma}_{r}^{2}}{2 \kappa^{2}} .
\end{aligned}
$$

Therefore, when we introduce a multiscale short rate we find that the calculation of the CDO prices is modified in two ways: i) the correction for the survival probabilities involve now $\mathcal{O}(1)$ and $\mathcal{O}(n)$ terms as announced above, ii) the discounting factor is modified as:

$$
e^{-r T} \mapsto e^{-\left[\bar{\theta}_{r, \infty}(T-B(T))+\left[1+n \rho_{r}\right] \bar{\sigma}_{r}^{2} B^{2}(T) /(4 \kappa)+r_{0} B(T)\right]} .
$$

We finish this section with a numerical example illustrating short scale fluctuation effects, shown in Figure 3. The parameters are chosen as in the constant parameter case of Figure 2. In addition we choose $\sigma_{r}=.1, \kappa_{r}=.25, c_{0}=-.05$ and $c_{1}=-.0007$ to illustrate how multiscale short rate fluctuations may affect the tranches. Note that with these parameters the loss distribution is not much affected. However, term structure effects influence relatively strongly the mezzanine tranches. This mechanism is somewhat complementary to the modification seen in the previous examples.

\section{$5 \quad$ Name Heterogeneity}

We return now to a non-symmetric model and describe how we can effectively compute the CDO price in this situation. We consider the case with one volatility factor:

$$
d X_{t}^{(i)}=\kappa\left(\theta_{i}-X_{t}^{(i)}\right) d t+v_{i} f\left(Y_{t}\right) d W_{t}^{(i)},
$$



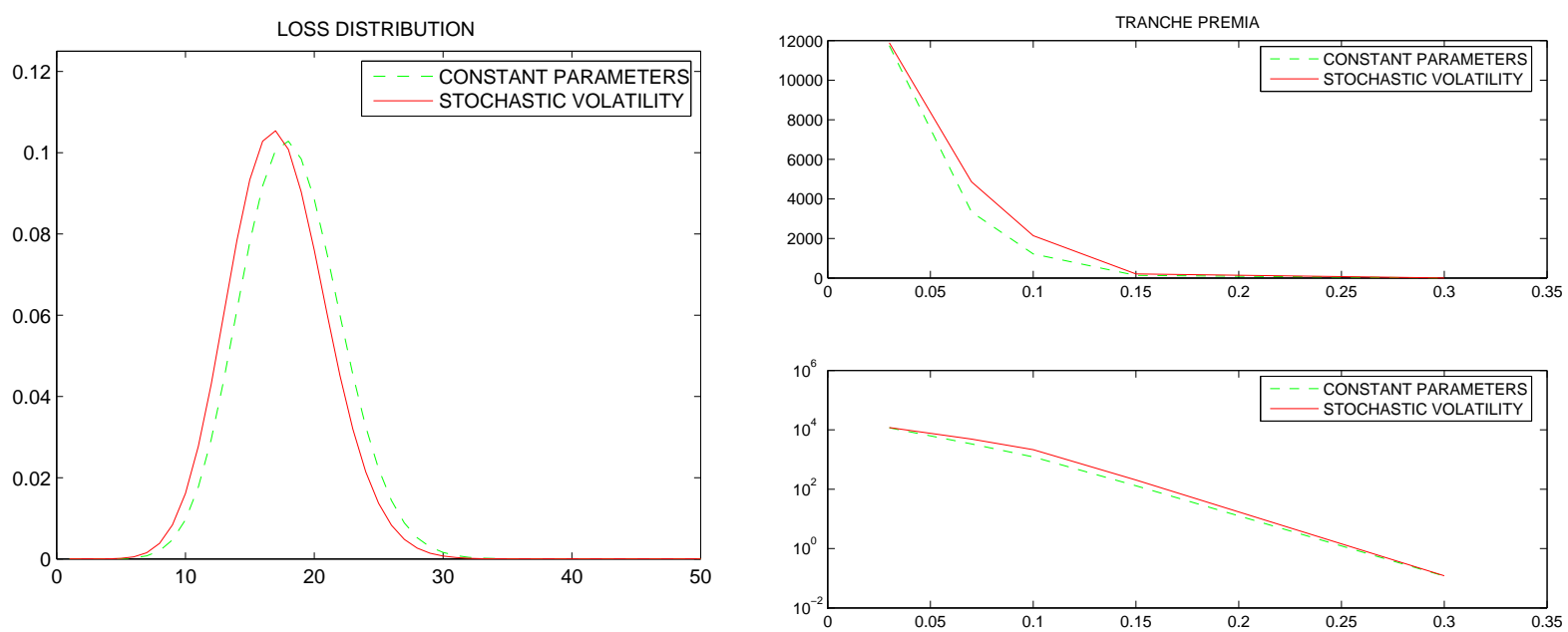

Figure 3: Loss distribution and tranche prices without and with short rate stochastic volatility effects.

for $1 \leq i \leq N$ and with $v_{i}$ ad $\theta_{i}$ constants. Moreover, the $W^{(j)}$ 's are correlated Brownian motions as in (2) and

$$
d Y_{t}=\frac{1}{\varepsilon}\left(m-Y_{t}\right) d t+\frac{\nu \sqrt{2}}{\sqrt{\varepsilon}} d W_{t}^{(y)}
$$

with again the symmetric correlations:

$$
\begin{aligned}
& d\left\langle W^{(i)}, W^{(y)}\right\rangle_{t}=\rho_{Y} d t, \quad \text { for } \quad 1 \leq i \leq N \\
& d\left\langle W^{(i)}, W^{(j)}\right\rangle_{t}=\rho_{X} d t, \quad \text { for } \quad i \neq j .
\end{aligned}
$$

Thus, we assume that the market is characterized by the two time constants $1 / \kappa$ and $1 / \varepsilon$, moreover, that the volatilities dependence on the fast factor is via the common term $f(Y)$. Therefore, in terms of their time scale contents the names are symmetric. We shall see below that this means that we can compute the CDO prices effectively via conditioning on, and subsequent integration with respect to, one Gaussian random variable. In the case that there are several time constants, several $\kappa$ 's characterizing the market, the CDO can be computed via conditioning with respect to several Gaussian random variables as we describe below.

The approximation for the joint survival probability can now be expressed by:

$$
\begin{aligned}
S(T ; \mathbf{x}, n) \sim(1+ & \left.v_{3}\left(\left(1-\rho_{X}\right) \sum_{i=1}^{n} \bar{\sigma}_{i} \sum_{i=1}^{n} \bar{\sigma}_{i}^{2}+\rho_{X}\left(\sum_{i=1}^{n} \bar{\sigma}_{i}\right)^{3}\right) B^{(3)}(T)\right) \\
& \times \tilde{A}^{c}(T) \prod_{i=1}^{n} \tilde{A}_{i}(T) e^{-B(T) x_{i}}
\end{aligned}
$$


where

$$
\begin{aligned}
\tilde{A}_{i}(T) & =e^{-\theta_{i} \kappa B^{(1)}(s)+\frac{1}{2} \bar{\sigma}_{i}^{2}\left(1-\rho_{X}\right) B^{(2)}(T)}, \\
\tilde{A}^{c}(T) & =e^{\left(\sum_{i=1}^{n} \bar{\sigma}_{i}\right)^{2}\left(\rho_{X} B^{(2)}(T) / 2\right)}, \\
\bar{\sigma}_{i}^{2} & =v_{i}^{2}\left\langle f^{2}(\cdot)\right\rangle, \\
B^{(k)}(T) & =\int_{0}^{T} B^{k}(s) d s .
\end{aligned}
$$

In order to compute the CDO price, we need the loss distribution $p_{n}(T ; \mathbf{x}, N)$ :

$$
p_{n}(T ; \mathbf{x}, N)=\mathbb{P}^{\star}\left\{(\# \text { names defaulted at time } T)=n \mid X_{0}=x_{i}\right\} .
$$

Consider first the case in which the names are independent:

$$
S(T ; \mathbf{x}, n)=\prod_{i=1}^{n} S_{i}\left(T ; x_{i}\right) .
$$

In this case we can compute the loss distribution via the recursive algorithm described in [12]. In this procedure the loss distribution for $n$ names is easily computed from the loss distribution of $n-1$ names due to independence, and so on. The procedure is $\mathcal{O}\left(n^{2}\right)$.

We now comment on how this procedure can be used to compute the loss distribution associated with the survival probabilities in (6). First, we discuss the case without stochastic volatility, but with correlation. Then we can write

$$
\begin{aligned}
S(T ; \mathbf{x}, n) & =\mathbb{E}\left\{\prod_{i=1}^{n} e^{Z \sum_{i=1}^{n} \sigma_{i} \sqrt{\rho_{X} B^{(2)}(T)}} \tilde{A}_{i}(T) e^{-B(T) x_{i}}\right\} \\
& =\mathbb{E}\left\{\prod_{i=1}^{n} \tilde{q}_{i}\left(T ; x_{i}\right)\right\},
\end{aligned}
$$

where the expectation is with respect to the standard Gaussian random variable $Z$ and we assume $\rho_{X} \geq 0$. The integration is therefore over cases corresponding to independent names, the situation in which the CDO price can be computed effectively by the iterative algorithm in [12]. Note that, as above, we constrain the Gaussian random variables so that $0 \leq \tilde{q}_{i} \leq 1$.

In Figure 4, we illustrate the effects of correlation in between the names and of hazard rate heterogeneity. We choose here $\rho_{X}=.3$, and we weight the first ten names' intensities by a factor of five, we keep the next fifty with with weight one, and the last 65 with weights 0.2 . The parameters are otherwise chosen as above. The result is shown by the solid line. The case without name correlation is shown by the dash-crossed line. The dashed line corresponds to replacing the heterogeneous hazard rate by its average. Note that this gives a very different loss distribution in this case with grouping of the hazard rate.

Now consider the case with both correlation and stochastic volatility. In order to extend the 

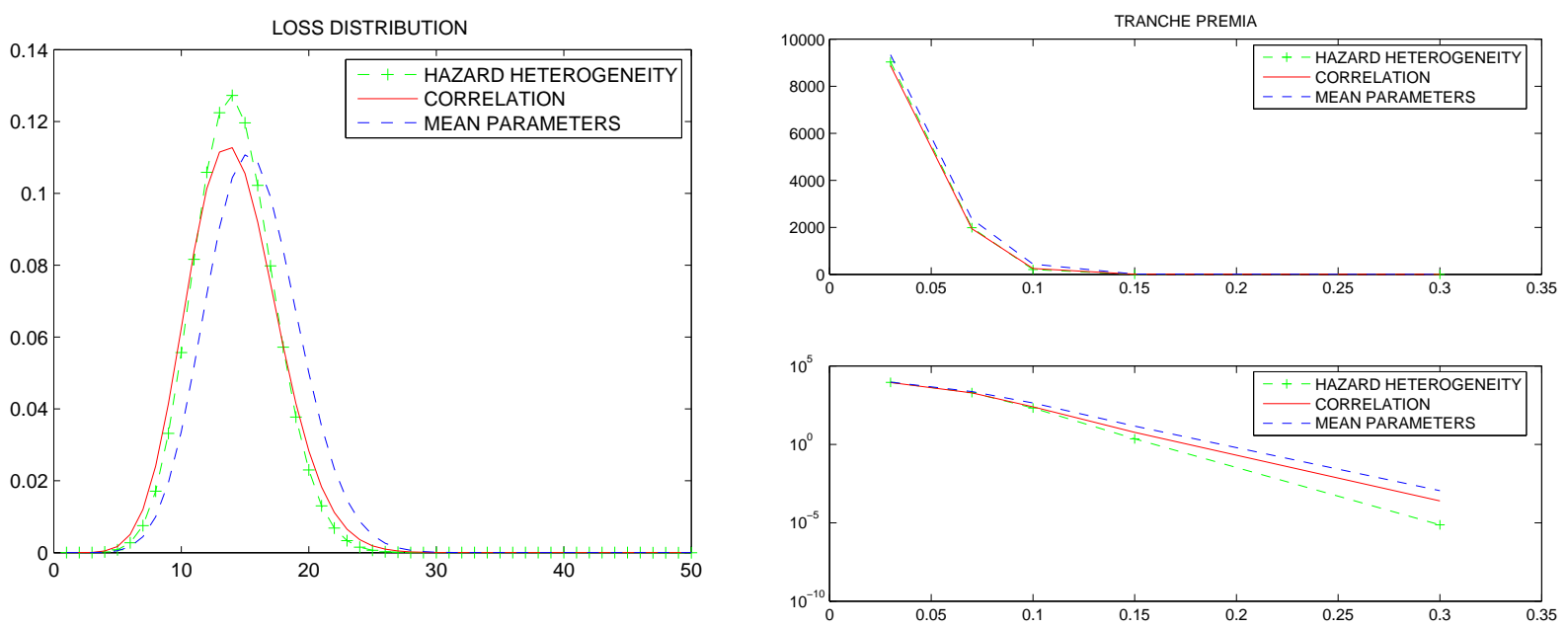

Figure 4: Loss distribution and tranche prices without and with correlation and heterogeneity effects.

calculation of the loss distribution to this case we use expansions of the form:

$$
\begin{aligned}
& \sqrt{\varepsilon}\left(\sum_{i=1}^{n} \bar{\sigma}_{i} \sum_{i=1}^{n} \bar{\sigma}_{i}^{2}\right)=\left(e^{\varepsilon^{3 / 8}\left(\sum_{i=1}^{n} \bar{\sigma}_{i}^{2}\right)}-1\right) \times\left\{e^{\varepsilon^{1 / 8}\left(\sum_{i=1}^{n} \bar{\sigma}_{i}\right)}\right. \\
& \left.-e^{\varepsilon^{1 / 4} / 2\left(\sum_{i=1}^{n} \bar{\sigma}_{i}\right)^{2}}-\left(e^{\varepsilon^{1 / 4}\left(\sum_{i=1}^{n} \bar{\sigma}_{i}\right)^{2}}-1\right) \times\left(e^{\varepsilon^{1 / 8}\left(\sum_{i=1}^{n} \bar{\sigma}_{i}\right)}-e^{\varepsilon^{1 / 4} / 2\left(\sum_{i=1}^{n} \bar{\sigma}_{i}\right)^{2}}\right)\right\}+\mathcal{O}\left(\varepsilon^{7 / 8}\right) \\
& \sqrt{\varepsilon}\left(\sum_{i=1}^{n} \bar{\sigma}_{i}\right)^{3}=\left(e^{\varepsilon^{3 / 8}\left(\sum_{i=1}^{n} \bar{\sigma}_{i}\right)^{2}}-1\right) \times\left\{e^{\varepsilon^{1 / 8}\left(\sum_{i=1}^{n} \bar{\sigma}_{i}\right)}\right. \\
& \left.-e^{\varepsilon^{1 / 4} / 2\left(\sum_{i=1}^{n} \bar{\sigma}_{i}\right)^{2}}-\left(e^{\varepsilon^{1 / 4}\left(\sum_{i=1}^{n} \bar{\sigma}_{i}\right)^{2}}-1\right) \times\left(e^{\varepsilon^{1 / 8}\left(\sum_{i=1}^{n} \bar{\sigma}_{i}\right)}-e^{\varepsilon^{1 / 4} / 2\left(\sum_{i=1}^{n} \bar{\sigma}_{i}\right)^{2}}\right)\right\}+\mathcal{O}\left(\varepsilon^{7 / 8}\right) .
\end{aligned}
$$

From (40) it then follows that we can express the loss distribution as a sum of terms of the form:

$$
S(T ; \mathbf{x}, n)=\sum_{k=1}^{K} a_{k} \mathbb{E}\left\{\prod_{i=1}^{n} e^{-c_{1, i, k}(T)+Z c_{2, k}(T) \bar{\sigma}_{i}-B(T) x_{i}}\right\},
$$

with $c_{1}>0$ and $c_{2}$ being $\mathcal{O}(1)$ and having $\mathcal{O}(\sqrt{\varepsilon})$ corrections due to stochastic volatility. Note that we have the normalization

$$
\sum_{k=1}^{K} a_{k}=1
$$

We can therefore again obtain the loss distribution via integration with respect to one Gaussian 
random variable over independent cases. This follows explicitly since using (43) we can write

$$
\begin{aligned}
p_{n}(T ; \mathbf{x}, N) & =\mathbb{P}^{\star}\{(\# \text { names defaulted at time } T)=n\} \\
= & \mathbb{E}^{\star}\left\{\sum_{\mathbf{s} \in \mathbf{S}_{n}}\left(\prod_{i \in \mathbf{s}^{c}} e^{-\int_{0}^{T} X_{t}^{(i)} d t} \prod_{i \in \mathbf{s}}\left(1-e^{-\int_{0}^{T} X_{t}^{(i)} d t}\right)\right) \mid X_{0}=\mathbf{x}\right\} \\
= & \mathbb{E}^{\star}\left\{\sum_{\mathbf{s} \in \tilde{\mathbf{S}}} c_{n}(\mathbf{s}) \prod_{i \in \mathbf{s}} e^{-\int_{0}^{T} X_{t}^{(i)} d t} \mid X_{0}=\mathbf{x}\right\} \\
& \sim \sum_{\mathbf{s} \in \tilde{\mathbf{S}}} c_{n}(\mathbf{s}) \sum_{k=1}^{K} a_{k} \mathbb{E}\left\{\prod_{i \in \mathbf{s}} e^{-c_{1, i, k}(T)+Z c_{2, k}(T) \bar{\sigma}_{i}-B(T) x_{i}}\right\} \\
= & \sum_{k=1}^{K} a_{k} \mathbb{E}\left\{\sum_{\mathbf{s} \in \tilde{\mathbf{S}}} c_{n}(\mathbf{s}) \prod_{i \in \mathbf{s}} e^{-c_{1, i, k}(T)+Z c_{2, k}(T) \bar{\sigma}_{i}-B(T) x_{i}}\right\}=\sum_{k=1}^{K} a_{k} \mathbb{E}\left\{\tilde{p}_{n}(T ; Z, \mathbf{x}, N)\right\} \\
= & \int \tilde{p}_{n}(T ; Z, \mathbf{x}, N) d \mu_{K}(Z),
\end{aligned}
$$

with $\mathbf{S}_{n}$ being the collection of distinct subsets of size $n$ of $\{1, \cdots, N\}, \tilde{\mathbf{S}}$ being all the distinct subsets or $\tilde{\mathbf{S}}=\mathbf{S}_{1} \cup \cdots \cup \mathbf{S}_{N}$ and with $\mathbf{s}^{c}$ being the complement set of $\mathbf{s}$. Observe that $\tilde{p}_{n}$ again can be computed effectively by the algorithm of the independent case as described in [12]. Moreover, $\mu_{K}$ is a signed measure with unit total mass. Indeed the loss distribution calculated by the above algorithm will therefore also have unit total mass. We remark that the possibility that the distribution can go negative can be dealt with via the framework set forth in [11].

We finally remark on the case with many time scales, that is with many different $\kappa_{i}$ 's. Note that we then can write

$$
\begin{aligned}
\log \left(\tilde{A}^{c}(T)\right) & =\frac{\rho_{X}}{2} \sum_{i, j=1}^{n} \bar{\sigma}_{j} \bar{\sigma}_{i} \int_{0}^{T} B\left(s ; \kappa_{i}\right) B\left(s ; \kappa_{j}\right) d s \\
& =\frac{\rho_{X}}{2} \sum_{i, j=1}^{n} \bar{\sigma}_{j} \bar{\sigma}_{i}\left(\frac{T}{\kappa_{i} \kappa_{j}}-B\left(T ; \kappa_{i}\right)-B\left(T ; \kappa_{j}\right)+B\left(T ; \kappa_{i}+\kappa_{j}\right)\right),
\end{aligned}
$$

with $B(t ; \kappa)=\left(1-e^{-\kappa t}\right) / \kappa$. Assume that there are at most $K$ distinct time scales $\kappa_{1}, \cdots, \kappa_{K}$. We denote by $A_{i j}$ the summands in (44). Then the matrix $\mathbf{A}=\left(A_{i j}\right)$ is symmetric non-negative definite and has rank at most $K$ so that in terms of the corresponding eigenvalue factorization we have

$$
\mathbf{A}=\sum_{i=1}^{K} \lambda_{i} \mathbf{v}^{(i)}\left(\mathbf{v}^{(i)}\right)^{T} .
$$

Using this fact we express

$$
\begin{aligned}
& S(T ; \mathbf{x}, n)=\mathbb{E}\left\{\prod_{i=1}^{n} \prod_{j=1}^{K} e^{\sqrt{2 \lambda_{j}} Z_{j} \sum_{i=1}^{n} v_{i}^{(j)}} S_{i}\left(T ; x_{i}\right)\right\} \\
& =\mathbb{E}\left\{\prod_{i=1}^{n} \prod_{j=1}^{K} e^{\sqrt{2 \lambda_{j}} Z_{j} v_{i}^{(j)}} S_{i}\left(T ; x_{i}\right)\right\},
\end{aligned}
$$

where the expectation is with respect to the independent standard Gaussian random variables $Z_{i}$. We remark that the spectrum $\mathbf{A}$ decays as $\left\{\left(\kappa_{i}+\kappa_{j}\right)^{-1}\right\}$, which typically has extremely fast decay. 
Thus, in constructing a numerical approximation for a CDO with high relative accuracy, we need only integrate with respect to a few eigenvalues.

We can decompose the terms in $D^{\varepsilon}$ similarly so that the CDO price in the case with many time scales, different $\kappa_{i}$ 's, can be obtained via integration with respect to a set of Gaussian random variables both in the case with and without stochastic volatility. In the case with stochastic volatility we integrate with respect to at most $K^{2}$ Gaussian random variables. This follows since we can write

$$
\begin{gathered}
\left\{\bar{\sigma}_{i} \bar{\sigma}_{j}^{2} \int_{0}^{T} B\left(s ; \kappa_{i}\right) B\left(s ; \kappa_{j}\right)^{2} d s\right\}_{i, j=1}^{N}=\sum_{i=1}^{K} \tilde{\sigma}_{i} \mathbf{u}_{i}\left(\mathbf{v}_{i}\right)^{T}, \\
\left\{\bar{\sigma}_{i} \bar{\sigma}_{j} \bar{\sigma}_{k} \int_{0}^{T} B\left(s ; \kappa_{i}\right) B\left(s ; \kappa_{j}\right) B\left(s ; \kappa_{k}\right) d s\right\}_{i, j=1}^{N}=\sum_{i=1}^{K^{2}} \tilde{\lambda}_{i, k} \tilde{\mathbf{v}}_{i}\left(\tilde{\mathbf{v}}_{i}\right)^{T},
\end{gathered}
$$

and then use decompositions as in (41) and (42).

The integration over the Gaussian random variables is again over cases corresponding to independent names, the situation in which the CDO price can be computed effectively by the iterative algorithm in [12]. As remarked above, if $K$ is large, one can typically obtain high relative accuracy using only a small part of the spectrum of the coupling matrices.

We finish this section with a numerical example. In Figures 5, we show the case when the stochastic volatility parameter $v_{3}=.001, v_{i}=2+2 \exp (-i / 120)$ and $\rho_{X}=0$. Note how the heterogeneity affects the loss distribution tail as shown in Figure 5. The parameters are chosen so that the loss distribution is almost zero before a small tail mode generated by implicit correlations. This gives a relatively large correction of the most senior tranche as shown in the the bottom right graph. We remark that if we replace $v_{i}$ by its simple average then there is no correction in this tranche price.
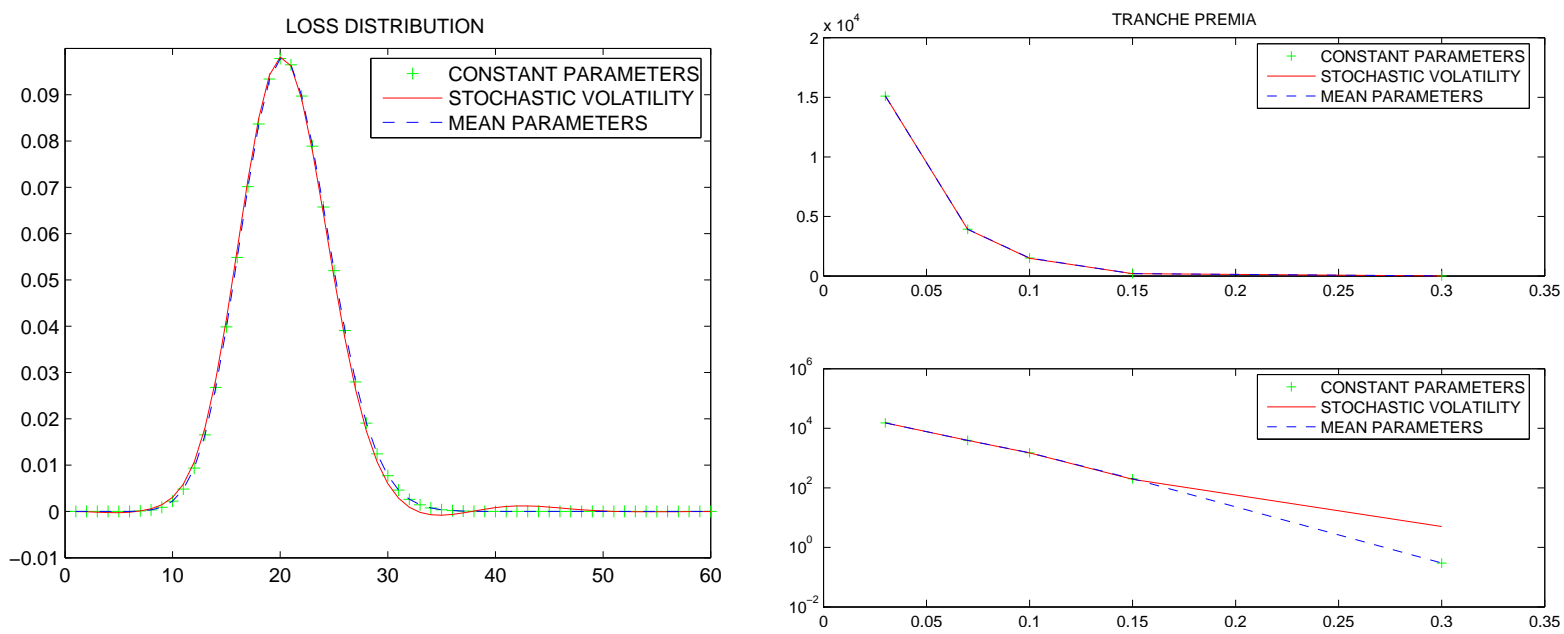

Figure 5: Stochastic volatility and name heterogeneity effects.

\section{Conclusions}

The results show that much progress can be made with multiscale stochastic volatility asymptotic approximations, even when built around a simple Vasicek-based model for stochastic intensities, 
by providing additional correlation through potentially large and rapid volatility excursions. Generalizing this approach for other popular intensity models such as CIR or expOU, while keeping the computational tractability, is an important challenge, which is work in progress. However, the Vasicek analysis provides insights into the relative roles of the model parameters, and their effects on loss distributions CDO tranche spreads. For example, the name-name correlation affects strongly the equity tranche. Uncertainty in the short rate affects relatively strongest the mezzanine tranches, while stochastic volatility in hazard gives a strong correction to senior tranches. Calibration to real data is an essential next step, which should be facilitated by explicit approximations computed here.

\section{References}

[1] P. Cotton, J.-P. Fouque, G. Papanicolaou, and K. R. Sircar. Stochastic volatility corrections for interest rate models. Mathematical Finance, 14(2):173-200, 2004.

[2] D. Duffie and N. Gârleanu. Risk and valuation of collateralized debt obligations. Financial Analysts Journal, 57(1):41-59, 2001.

[3] D. Duffie and K. Singleton. Credit Risk. Princeton University Press, 2003.

[4] A. Eckner. Computational techniques for basic affine models of portfolio credit risk. Technical report, Stanford University, 2007.

[5] A. Elizalde. Credit risk models IV: Understanding and pricing CDOs. www.abelelizalde.com, 2005.

[6] E. Errais, K. Giesecke, and L. Goldberg. Pricing credit from the top down with affine point processes. Working paper, Stanford University, February 2007.

[7] J.-P. Fouque, G. Papanicolaou, and R. Sircar. Derivatives in Financial Markets with Stochastic Volatility. Cambridge University Press, 2000.

[8] J.-P. Fouque, G. Papanicolaou, R. Sircar, and K. Solna. Multiscale stochastic volatility asymptotics. SIAM J. Multiscale Modeling \& Simulation, 2(1):22-42, 2003.

[9] J.-P. Fouque, R. Sircar, and K. Sølna. Stochastic volatility effects on defaultable bonds. Applied Mathematical Finance, 13(3):215-244, 2006.

[10] J.-P. Fouque, B.C. Wignall, and X. Zhou. Modeling correlated defaults: First passage model under stochastic volatility. Journal of Computational Finance, 11(3):43-78, 2008. To appear.

[11] J.-P. Fouque and X. Zhou. Perturbed gaussian copula. Advances in Econometrics, To appear, 2008.

[12] J. Hull and A. White. Valuation of a CDO and an n-th to default CDS without Monte Carlo simulation. Journal of Derivatives, 12(2):8-23, 2004.

[13] T. Hurd, A. Kuznetsov, and Z. Zhou. Credit risk using time changed Brownian motions. Technical report, McMaster University, 2008.

[14] A. Lopatin and T. Misirpashaev. Two-dimensional Markovian model for dynamics of aggregate credit loss. Technical report, Numerix, 2007.

[15] A. Mortensen. Semi-analytical valuation of basket credit derivatives in intensity-based models. Journal of Derivatives, 13(4):8-26, 2006.

[16] E. Papageorgiou and R. Sircar. Multiscale intensity models and name grouping for valuation of multiname credit derivatives. Submitted, 2007.

[17] P. Schönbucher. Credit Derivatives Pricing Models. Wiley, 2003.

[18] P. Schönbucher. Portfolio losses and the term structure of loss transition rates: A new methodology for the pricing of portfolio credit derivatives. Technical report, ETH Zurich, 2006. 